\title{
Low-temperature specific heat of rare-earth-doped silicate glasses
}

\author{
D. A. van de Straat, J. Baak, and H. B. Brom \\ Kamerlingh Onnes Laboratorium, Leiden University, P.O. Box 9506, 2300RA Leiden, The Netherlands \\ Th. Schmidt* and S. Völker \\ Huygens Laboratorium, Leiden University, P.O. Box 9504, 2300 RA Leiden, The Netherlands
}

(Received 31 July 1995)

\begin{abstract}
The specific heat $C_{v}$ of $\mathrm{Pr}^{3+}$ - and $\mathrm{Eu}^{3+}$-doped silicate glasses has been determined between 0.1 and $10 \mathrm{~K}$. Below about $5 \mathrm{~K}, C_{v} \propto T^{1+\nu}$ with $\nu=0.0-0.3$. This dependence is characteristic for the two-level-system (TLS) contribution to the specific heat, $C_{v}^{\mathrm{TLS}}$. The values of $C_{v}^{\mathrm{TLS}}$ and $\nu$ noticeably vary with the chemical nature and the concentration of the glass modifiers. The number of TLS in samples of almost identical composition is significantly larger for the $\operatorname{Pr}^{3+}$ doped than for the $\mathrm{Eu}^{3+}$ doped and the undoped glass. Above $5 \mathrm{~K}$, the $T^{3}$-phonon term and the $T^{5}$ term associated with soft localized vibrations also contribute to $C_{v}$. The results do not point to a correlation between the $T^{1+\nu}$ and the higher-order terms.
\end{abstract}

The existence of a quasilinear contribution to the lowtemperature specific heat of glasses ${ }^{1-3}$ is usually explained by so-called two-level systems (TLS). ${ }^{4,5}$ It is assumed that these TLS are (groups of) atoms or molecules tunneling from one spatial position to another. ${ }^{1,2,4-8}$ Recently, intensity fluctuations in the autocorrelation of the fluorescence of single aromatic molecules in polyethylene at $2 \mathrm{~K}$ have been interpreted as tunneling of individual TLS. ${ }^{9}$ Furthermore, we have found for two different silicate glasses that both the specific heat and the optical linewidth follow a quasilinear, $T^{1+\nu}$, temperature dependence, with the same value of $\nu$ for a given rare-earth/glass sample, but with different $\nu$ for the two samples. ${ }^{10}$ In the present work we show that the lowtemperature specific heat depends on the composition of the silicate glass and that, for a glass with a given composition, the number of TLS is significantly enhanced when doped with $\operatorname{Pr}^{3+}$ as compared to $\mathrm{Eu}^{3+}$ and the undoped glass.

Five samples with dimensions of approximately $0.5 \mathrm{~cm}$ $\times 0.5 \mathrm{~cm} \times 1 \mathrm{~cm}$ were used. The rare-earth ions $\operatorname{Pr}^{3+}$ and $\mathrm{Eu}$ ${ }^{3+}$ were doped at different concentrations in silicate glasses of varying composition (see Table I). ${ }^{11}$ The first three glasses in the table have identical glass modifiers with very similar concentrations and differ only in the dopants. The fourth sample doped with $1 \mathrm{~mol} \% \operatorname{Pr}^{3+}$ has the same glass modifiers as the first three but with different concentrations. The last sample doped with $0.25 \mathrm{~mol} \% \mathrm{Eu}^{3+}$, in addition, differs in the composition of the glass modifiers. The names in Table I refer to the concentration of the rare-earth ion, which is given in mol \%.

The specific-heat data were obtained in a dilution refrigerator using a thermal relaxation method. ${ }^{12}$ In this method the sample is permanently linked to a heat sink. Averaged temperature profiles are obtained by periodic applications of a heat pulse. The heat capacity follows from the power applied, from the equilibrium temperature reached during the heat pulse and from the area of the temperature profile in a $T$-versus-time plot. ${ }^{13}$ The sample was thermally connected to the top of a sapphire plate with dimensions $1 \mathrm{~cm} \times 0.7 \mathrm{~cm}$ $\times 0.1 \mathrm{~cm}$ by a precisely determined amount of Apiezon- $\mathrm{N}$ grease. A ruthenium-oxide resistor was used as thermometer. A NiCr heater with a resistance of about $1 \mathrm{k} \Omega$ was sputtered in a meandering pattern on the bottom of the sapphire plate. A small gold wire with a length of about $5 \mathrm{~cm}$ and a diameter of $40 \mu \mathrm{m}$ provided the heat link between sample holder and heat sink. The calculated heat capacity was corrected for the contributions of the empty apparatus (measured in a separate experiment) and the amount of Apiezon-N grease. ${ }^{14-16}$ The heating time varied between $15 \mathrm{~s}$ and $400 \mathrm{~s}$, i.e., the experiments were performed in the long-time regime. ${ }^{17}$

The data of the specific heat $C_{v}$ as a function of temperature $T$ between about 0.1 and $10 \mathrm{~K}$ for the three samples of almost identical silicate glass composition $(R 0.0, \operatorname{Pr} 0.1$, and Eu 3.0) are presented in Fig. 1(a). The specific-heat data for the last two samples of Table I, Pr 1.0 and Eu 0.25, are shown in Figs. 1(b) and 1(c) together with those of Pr 0.1 and $\mathrm{Eu} 3.0$, respectively, for comparison. The curves through the data are fits to the following expression:

$$
\begin{aligned}
C_{v} & =C_{v}^{\mathrm{TLS}}+C_{v}^{D}+C_{v}^{\mathrm{loc}} \\
& =a_{\mathrm{TLS}} T^{1+\nu}+\left(a_{D}+a_{\mathrm{exc}}\right) T^{3}+a_{\mathrm{loc}} T^{5} .
\end{aligned}
$$

The first term, $C_{v}^{\mathrm{TLS}}=a_{\mathrm{TLS}} T^{1+\nu}$, represents the TLS contribution to the specific heat, where the parameter $\nu$ is related to the energy dependence of the TLS density of states, $\rho=\rho_{0} E^{\nu},{ }^{18}$ with $\nu=0-0.3$. ${ }^{1,3}$ The second term $C_{v}^{D}$ is a $T^{3}$-phonon contribution consisting of two parts, the Debye part, $a_{D} T^{3}$, related to the elastic constants, and an additional "excess" term characteristic for glasses, $a_{\text {exc }} T^{3}$, which is usually of the same order as the Debye term., ${ }^{3,7,17}$ The third term, proportional to $T^{5}$, accounts for the broad plateau often observed in $C_{v} / T^{3}$ plots in the region of 5-10 K. ${ }^{1,2,19-22}$ The fitting parameters $\left(\nu, a_{\mathrm{TLS}}, a_{D}+a_{\mathrm{exc}}, a_{\mathrm{loc}}\right)$ are given in Table II. For comparison, the values of the pure silicate glass Suprasil-W, taken from the literature, ${ }^{3}$ are also given.

We can estimate the number of TLS, $N_{\text {TLS }}$, that contributes to the specific heat in a selected temperature interval from the entropy difference: ${ }^{23}$

$$
\Delta S=N_{\mathrm{TLS}} k_{B} \ln 2=\int_{0}^{T_{\max }}\left(C_{v}^{\mathrm{TLS}} / T\right) d T,
$$


TABLE I. Compositions of the rare-earth-doped silicate glass samples.

\begin{tabular}{|c|c|c|}
\hline Name & Comp. & Mol \% (Ref. 11) \\
\hline \multirow[t]{4}{*}{ R 0.0} & $\mathrm{SiO}_{2}$ & 60 \\
\hline & $\mathrm{Li}_{2} \mathrm{O}$ & 27.5 \\
\hline & $\mathrm{CaO}$ & 10.0 \\
\hline & $\mathrm{Al}_{2} \mathrm{O}_{3}$ & 2.5 \\
\hline \multirow[t]{5}{*}{$\operatorname{Pr} 0.1$} & $\mathrm{SiO}_{2}$ & 59.9 \\
\hline & $\mathrm{Li}_{2} \mathrm{O}$ & 27.5 \\
\hline & $\mathrm{CaO}$ & 10 \\
\hline & $\mathrm{Al}_{2} \mathrm{O}_{3}$ & 2.5 \\
\hline & $\mathrm{Pr}_{2} \mathrm{O}_{3}$ & 0.1 \\
\hline \multirow[t]{5}{*}{ Eu 3.0} & $\mathrm{SiO}_{2}$ & 57 \\
\hline & $\mathrm{Li}_{2} \mathrm{O}$ & 27.5 \\
\hline & $\mathrm{CaO}$ & 10.0 \\
\hline & $\mathrm{Al}_{2} \mathrm{O}_{3}$ & 2.5 \\
\hline & $\mathrm{Eu}_{2} \mathrm{O}_{3}$ & 3.0 \\
\hline \multirow[t]{5}{*}{$\operatorname{Pr} 1.0$} & $\mathrm{SiO}_{2}$ & 68.3 \\
\hline & $\mathrm{Li}_{2} \mathrm{O}$ & 15 \\
\hline & $\mathrm{CaO}$ & 11 \\
\hline & $\mathrm{Al}_{2} \mathrm{O}_{3}$ & 4.7 \\
\hline & $\mathrm{Pr}_{2} \mathrm{O}_{3}$ & 1.0 \\
\hline \multirow[t]{5}{*}{ Eu 0.25} & $\mathrm{SiO}_{2}$ & 74.75 \\
\hline & $\mathrm{Na}_{2} \mathrm{O}$ & 15 \\
\hline & $\mathrm{BaO}$ & 5 \\
\hline & $\mathrm{ZnO}$ & 5 \\
\hline & $\mathrm{Eu}_{2} \mathrm{O}_{3}$ & 0.25 \\
\hline
\end{tabular}

where the upper cutoff temperature $T_{\max }$ can be rather arbitrarily chosen because the density of energy splittings, $E$, of the TLS is rather flat. ${ }^{18}$ We have chosen $T_{\text {max }}=5 \mathrm{~K}$ because optical dephasing experiments on the Pr 0.1 and the Eu 0.25 samples have suggested the presence of TLS up to at least this temperature. ${ }^{10}$ With $C_{v}^{\mathrm{TLS}}=a_{\mathrm{TLS}} T^{1+\nu}$, we obtain

$$
N_{\mathrm{TLS}}=\frac{a_{\mathrm{TLS}} 5^{1+\nu}}{\left[k_{B}(1+\nu) \ln 2\right]} \text {. }
$$

The results for $N_{\text {TLS }}$ are given in Table II. By comparing the five glassy samples studied, we see that $N_{\text {TLS }}$ depends strongly on the composition of the glass, i.e., on the glass modifiers and their mol \%, and on the rare-earth ions.

Figure 1(a) shows that of the three comparable silicate glasses, $R 0.0$ and Eu 3.0 have very similar values of the specific heat, whereas $\operatorname{Pr} 0.1$ has a significantly higher value of $C_{v}$. This is also shown in Table II, where $N_{\text {TLS }}$ is almost three times larger for $\operatorname{Pr} 0.1$ than for the other two samples. Furthermore, $N_{\text {TLS }}$ increases only slightly by doping the glass with $\mathrm{Eu}^{3+}$ (compare $R 0.0$ with $\mathrm{Eu} 3.0$ ). We attribute the significantly larger value of $N_{\text {TLS }}$ to the larger ionic radius of $\operatorname{Pr}^{3+}(r=1.08 \AA)$ compared to that of $\mathrm{Eu}^{3+}$ $(r=1.00 \AA) .{ }^{24}$ The influence of the rare-earth size is also

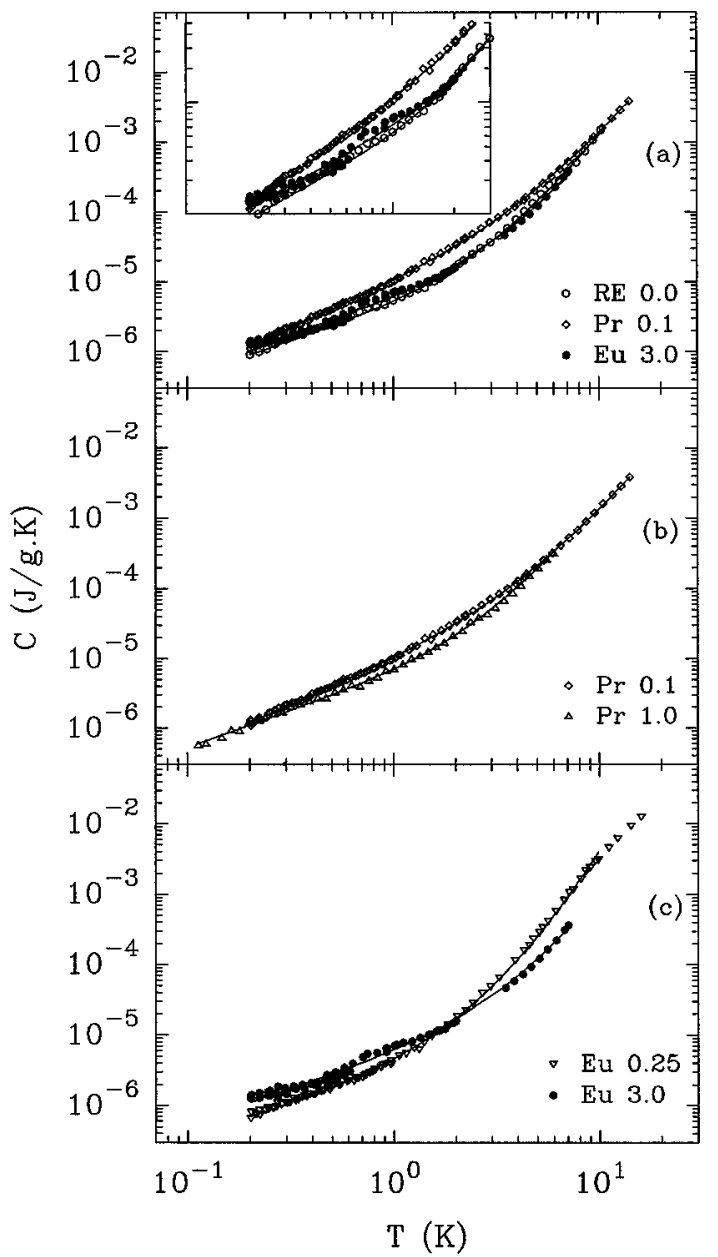

FIG. 1. Specific heat of the silicate glasses listed in Table I: (a) undoped glass $(R 0.0)(\bigcirc), \operatorname{Pr} 0.1(\diamond)$, and $3.0(\bullet)$, (b) $\operatorname{Pr} 1.0$ $(\triangle)$ and $\operatorname{Pr} 0.1(\diamond),(\mathrm{c})$ Eu $0.25(\nabla)$ and Eu $3.0(\bullet)$. Drawn lines are fits of Eq. (1) to the data with the parameter values given in Table II. The inset of (a) is a vertical enlargement of the lowtemperature data between 0.1 and $3 \mathrm{~K}$.

manifested in the orthorhombic high- $T_{c}$ superconducting compounds of the type $X \mathrm{Ba}_{2} \mathrm{Cu}_{3} \mathrm{O}_{7}$. For $X=\mathrm{Pr}^{3+}$ the compound is not superconducting, whereas it is for most other rare-earth ions. $^{25,26}$

The value of $N_{\text {TLS }}$ for $R 0.0$ is about two and a half times larger than that for the pure silicate glass Suprasil-W $(\approx$ $100 \% \mathrm{SiO}_{2}$ ), which proves that the presence of glass modifiers indeed increases the number of TLS. In Fig. 1(b), where the temperature dependence of the specific heat of $\operatorname{Pr} 0.1$ is compared to that of $\operatorname{Pr} 1.0$, we observe that a change in the concentration $(\mathrm{mol} \%)$ of the glass modifiers (see Table I) influences the value of $N_{\text {TLS }}$ more than the difference in $\mathrm{Pr}^{3+}$ concentration between 1.0 and $0.1 \%$. Figure 1(c) shows data for $\mathrm{Eu} 0.25$ and $\mathrm{Eu} 3.0$ which confirm the idea that the composition of the glass, which is markedly different for the two samples, strongly influences $N_{\mathrm{TLS}}$. It is therefore understandable that the Eu 0.25 sample yields a lower $N_{\text {TLS }}$ value than the $R 0.0$ sample.

We will only comment briefly on the $T^{3}$ and $T^{5}$ contributions to the specific heat, since they are not the main subjects 
TABLE II. Parameters obtained from the fits of the expression $C_{v}=a_{\mathrm{TLS}} T^{1+\nu}+\left(a_{D}+a_{\mathrm{exc}}\right) T^{3}+a_{\mathrm{loc}} T^{5}$ to the data. The number of TLS, $N_{\mathrm{TLS}}$, was calculated from the quasilinear contribution to the specific heat [see Eq. (3)].

\begin{tabular}{|c|c|c|c|c|c|}
\hline Sample & $\nu$ & $\begin{array}{c}a_{\mathrm{TLS}} \\
\frac{10^{-6} \mathrm{~J}}{\mathrm{~g} \mathrm{~K}^{2+\nu}}\end{array}$ & $\begin{array}{l}a_{D}+a_{\mathrm{exc}} \\
\frac{10^{-6} \mathrm{~J}}{\mathrm{~g} \mathrm{~K}^{4}}\end{array}$ & $\begin{array}{c}\begin{array}{c}a_{\mathrm{loc}} \\
10^{-9} \mathrm{~J}\end{array} \\
\mathrm{~g} \mathrm{~K}^{6}\end{array}$ & $\begin{array}{c}N_{T L S}(T<5 \mathrm{~K}) \\
\frac{10^{18} \mathrm{TLS}}{\mathrm{g}}\end{array}$ \\
\hline Suprasil- $W$ & $0.30 \pm 0.03$ (Ref. 3) & $1.5 \pm 0.1$ & & & $1.0 \pm 0.1$ \\
\hline$R 0.0$ & $0.00 \pm 0.04$ & $4.5 \pm 0.2$ & $0.8 \pm 0.1$ & $4 \pm 1$ & $2.4 \pm 0.2$ \\
\hline $\operatorname{Pr} 0.1$ & $0.29 \pm 0.02($ Ref. 10$)$ & $9.6 \pm 0.1$ (Ref. 10) & $1.1 \pm 0.1$ (Ref. 10) & $1 \pm 0.3$ (Ref. 10$)$ & $6.2 \pm 0.2$ \\
\hline $\mathrm{Eu} 3.0$ & $0.00 \pm 0.06$ & $5.6 \pm 0.2$ & $0.7 \pm 0.1$ & $3 \pm 1$ & $2.9 \pm 0.2$ \\
\hline $\operatorname{Pr} 1.0$ & $0.05 \pm 0.05$ & $6.0 \pm 0.2$ & $1.2 \pm 0.1$ & $5 \pm 2$ & $3.2 \pm 0.2$ \\
\hline Eu 0.25 & $0.01 \pm 0.02($ Ref. 10$)$ & $3.5 \pm 0.1$ (Ref. 10) & $1.2 \pm 0.1($ Ref. 10$)$ & $30 \pm 3($ Ref. 10) & $1.8 \pm 0.1$ \\
\hline
\end{tabular}

of this paper. The experimental values $\left(a_{D}+a_{\text {exc }}\right) \approx 0.8-$ 1.2 (see Table II) are about 1.2-1.5 times larger than the theoretical values $(\approx 0.7-0.8)$ expected from the Debye phonon contribution, $C_{v}^{D} / T^{3}=2 V k_{B}^{4} \pi^{2} /\left(5 v^{3} \hbar^{3}\right)$ in which $V$ is the specific volume and $v$ is the velocity of sound. ${ }^{23}$ The origin of this excess $T^{3}$-dependent specific heat, peculiar to the amorphous state and well documented in the literature, ${ }^{1,17,27-30}$ is still unknown.

The fitting coefficient $a_{\text {loc }}$ of the $T^{5}$ term, which in the literature is interpreted either as due to soft localized vibrations ${ }^{19,20,22}$ or to the onset of phonon dispersion, ${ }^{21}$ is much smaller than $a_{\mathrm{TLS}}$. It varies between $\sim 10^{-2}$ and $10^{-4} \times a_{\mathrm{TLS}}$, depending on the glass composition (see Table II). There appears to be no correlation between the $T^{1+\nu}$ and the higher-order terms when comparing different samples and, thus, we have no indication of a common origin of the TLS states and soft vibrations. ${ }^{8,22}$
In summary, we conclude from these low-temperature specific-heat experiments that the number of TLS in silicate glasses varies strongly with the chemical composition of the glass and, for a given composition, with the nature of the rare-earth dopant. $\operatorname{Pr}^{3+}$ induces a larger number of TLS than $\mathrm{Eu}^{3+}$, probably due to its larger size. In the present samples, the exponent $\nu$ ranges between 0.0 and 0.30 , implying that the energy splitting dependence of the low-temperature density of TLS states varies for silicate glasses of different compositions. Our experiments provide no evidence for a common origin of TLS states and soft vibrations.

We would like to thank Y. E. Volokitin for his help with the measurements, and R. M. Macfarlane, B. Jacquier, and M. J. Weber for generously providing us with the samples. Further we thank R. Silbey for critical comments and valuable remarks regarding the manuscript.
*Present address: Institute for Biophysics, Johannes-Kepler University, Altenberger Strasse 69, 4040 Linz, Austria.

${ }^{1}$ W.A. Phillips, Rep. Prog. Phys. 50, 1657 (1987), and references therein; Amorphous Solids Low-Temperature Properties, Topics in Current Physics Vol. 24, edited by W.A. Phillips (Springer, Berlin, 1981), and references therein.

${ }^{2}$ R.C. Zeller and R.O. Pohl, Phys. Rev. B 4, 2029 (1971).

${ }^{3}$ J.C. Lasjaunias, A. Ravex, M. Vandorpe, and S. Hunklinger, Solid State Commun. 17, 1045 (1975).

${ }^{4}$ P.W. Anderson, B.I. Halperin, and C.M. Varma, Philos. Mag. 25, 1 (1972).

${ }^{5}$ W.A. Phillips, J. Low Temp. Phys. 7, 351 (1972).

${ }^{6}$ A. Heuer and R.J. Silbey, Phys. Rev. Lett. 70, 3911 (1993); Phys. Rev. B 48, 9411 (1993); 49, 1441 (1994).

${ }^{7}$ J. Zimmermann and G. Weber, Phys. Rev. Lett. 46, 661 (1981).

${ }^{8}$ J.M. Grace and A.C. Anderson, Phys. Rev. B 40, 1901 (1989).

${ }^{9}$ A. Zumbusch, L. Fleury, R. Brown, J. Bernard, and M. Orrit, Phys. Rev. Lett. 70, 3584 (1993).

${ }^{10}$ Th. Schmidt, J. Baak, D.A. van de Straat, H.B. Brom, and S. Völker, Phys. Rev. Lett. 71, 3031 (1993).

${ }^{11}$ R.M. Macfarlane, B. Jacquier, and M.J. Weber (private communication).

${ }^{12}$ R. Bachman et al., Rev. Sci. Instrum. 43, 205 (1972).
${ }^{13}$ J. Baak et al., Physica C 168, 363 (1990).

${ }^{14}$ A.J. Bevolo, Cryogenics 14, 661 (1974).

${ }^{15}$ M. Wun and N. E. Phillips, Cryogenics 15, 36 (1975).

${ }^{16}$ H.J. Schink and H.v. Lohneysen, Cryogenics 21, 591 (1981).

${ }^{17}$ M.T. Loponen, R.C. Dynes, V. Narayanamurti, and J.P. Garno, Phys. Rev. Lett. 45, 457 (1980).

${ }^{18} \mathrm{In}$ order to get the general expression for $C_{v}^{\mathrm{TLS}}$ one has to take into account that the energy splittings $E$ of the TLS are not equal, but spread with a density function $\rho(E)$. At temperatures where the contribution of the TLS to $C_{v}$ is relevant, $\rho(E)=\rho_{0} E^{\nu}$, with $0<\nu<0.3$ (Ref. 1). The contribution of all TLS to the specific heat then becomes $C_{v}^{\mathrm{TLS}}=\int_{0}^{E_{\max }} \rho(E) C_{1}^{\mathrm{TLS}}(E) d E$, where $E_{\max }$ is the upper cutoff energy splitting, and the specific heat of a single TLS is given by $C_{1}^{\mathrm{TLS}}(E, T)=k_{B}\left(E / 2 k_{B} T\right)^{2} \operatorname{sech}^{2}\left(E / 2 k_{B} T\right)$ (Ref. 23). At low temperatures, $E_{\max } / 2 k_{B} T \gg 1$, the integral becomes analytically solvable: (Ref. 31$)$ :

$$
C_{v}^{\mathrm{TLS}}=\left(1-2 / 2^{\nu+2}\right) \Gamma(3+\nu) \zeta(2+\nu) \rho_{0} k_{B}^{2+\nu} T^{1+\nu},
$$

where $\Gamma(x)$ represents the gamma function, and $\zeta(x)$ the Riemann zeta function. At high temperatures, $T>E_{\max } / 2 k_{B}, C_{v}^{\mathrm{TLS}}$ is proportional to $1 / T^{2}$.

${ }^{19}$ L. Gil, M.A. Ramos, A. Bringer, and U. Buchenau, Phys. Rev. Lett. 70, 182 (1993); U. Buchenau, Europhys. News 24, 77 (1993). 
${ }^{20}$ U. Buchenau, Yu.M. Galperin, V.M. Gurevich, and H.R. Schober, Phys. Rev. B 43, 5039 (1991).

${ }^{21}$ H. von Löhneisen, H. Rüsing, and W. Sander, Z. Phys. B 60, 323 (1985).

${ }^{22}$ X. Liu and H. von Löhneisen, Phys. Rev. B 48, 13486 (1993).

${ }^{23}$ C. Kittel, Introduction to Solid State Physics (Wiley, New York, 1986).

${ }^{24}$ F. Gordon Smith, Physical Geochemistry (Addison-Wesley, Reading, MA, 1963).
${ }^{25}$ J.J. Neumeier, M.B. Maple, and M.S. Torikachvili, Physica C 156, 574 (1988).

${ }^{26}$ V.A. Voloshin, Physica C 168, 506 (1990).

${ }^{27}$ M. Meissner and K. Spitzmann, Phys. Rev. Lett. 46, 265 (1981).

${ }^{28}$ J. Zimmermann and G. Weber, Phys. Lett. 86A, 32 (1981).

${ }^{29}$ C.M. Varma, R.C. Dynes, and J.R. Banavar, J. Phys. C 15, L1221 (1982).

${ }^{30}$ C.C. Yu and J.J. Freeman, Phys. Rev. B 36, 7620 (1987).

${ }^{31}$ H.B. Dwight, Tables of Integrals and Other Mathematical Data, 4th ed. (MacMillan, New York, 1961), p. 234. 